\title{
FULL DUALITY FOR COACTIONS OF DISCRETE GROUPS
}

\author{
SIEGFRIED ECHTERHOFF and JOHN QUIGG*
}

\begin{abstract}
Using the strong relation between coactions of a discrete group $G$ on $C^{*}$-algebras and Fell bundles over $G$ we prove a new version of Mansfield's imprimitivity theorem for coactions of discrete groups. Our imprimitivity theorem works for the universally defined full crossed products and arbitrary subgroups of $G$ as opposed to the usual theory of [16], [11] which uses the spatially defined reduced crossed products and normal subgroups of $G$. Moreover, our theorem factors through the usual one by passing to appropriate quotients. As applications we show that a Fell bundle over a discrete group is amenable in the sense of Exel [7] if and only if the double dual action is amenable in the sense that the maximal and reduced crossed products coincide. We also give a new characterization of induced coactions in terms of their dual actions.
\end{abstract}

\section{Introduction}

One of the main tools in the study of crossed products by $C^{*}$-dynamical systems is Green's imprimitivity theorem $([9, \S 2])$ : starting with an action $\alpha: G \rightarrow \operatorname{Aut}(A)$ and a closed subgroup $H$ of $G$, it provides a Morita equivalence between the crossed products

$$
C_{0}(G / H, A) \times_{\tau \otimes \alpha} G \quad \text { and } \quad A \times_{\alpha \mid} H,
$$

where $\tau$ denotes the translation action of $G$ on $G / H$. Note that we can take either the full or the reduced crossed products in the formulation of Green's theorem (see [13, Theorem 3.15] and [19, Lemma 4.1] for the reduced versions). If $G$ is abelian, the imprimitivity theorem combined with Takesaki-Takai duality theory provides an even more powerful tool for the investigation of the structure of certain crossed products. Note that the connection of both theories is given by the existence of a natural isomorphism

$$
C_{0}(G / H, A) \times_{\tau \otimes \alpha} G \cong\left(A \times_{\alpha} G\right) \times_{\widehat{\alpha} \mid} \widehat{G / H},
$$

${ }^{*}$ Research partially supported by National Science Foundation Grant DMS9401253 and Deutsche Forschungsgemeinschaft (SFB 478)

Received July 1, 1999; in revised form November 19, 1999. 
where $\widehat{\alpha}$ denotes the dual action of the Pontrjagin dual $\widehat{G}$ on $A \times_{\alpha} G$ (e.g., see $[9, \S 7]$ and [1], [3] for details and applications). If $G$ is nonabelian, TakesakiTakai duality theory becomes a duality between actions and coactions of $G$, so in order to make these techniques available for the study of crossed products by actions and coactions of nonabelian groups it is necessary to have good working analogues of the imprimitivity theorem for coactions of groups on $C^{*}$-algebras.

A major step towards a general imprimitivity theorem for coactions was first achieved by Mansfield in [16], where he showed that, if $\delta: A \rightarrow M(A \otimes$ $C^{*}(G)$ ) is a coaction of $G$ on $A$ and $N$ is a normal and amenable closed subgroup of $G$, there exists a natural Morita equivalence between

$$
\left(A \times_{\delta} G\right) \times \times_{\widehat{\delta} \mid} N \quad \text { and } \quad A \times_{\delta \mid} G / N,
$$

where $\widehat{\delta}: G \rightarrow \operatorname{Aut}\left(A \times{ }_{\delta} G\right)$ denotes the dual action of $G$ on $A \times_{\delta} G$. This result was generalized to possibly non-amenable normal subgroups by the second author and Kaliszewski in [11], but with a technical restriction which is satisfied in particular when $\delta$ is a normal coaction in the sense of [17, Definition 2.1]. For such coactions they obtained a natural Morita equivalence for the reduced crossed product $\left(A \times_{\delta} G\right) \times \times_{\widehat{\delta} \mid, r} N$ and $A \times_{\delta \mid} G / N$. Since every coaction $\delta$ has a normalization (see [17, §2]), this result should be interpreted as a general analogue of Green's theorem for reduced crossed products (note that, if $N$ is amenable, $B \times{ }_{\beta} N \cong B \times_{\beta, r} N$ for every action $\beta$ of $N$, which explains why Mansfield's result can be regarded as a special case of the results in [11]).

In [4] it is shown that if we start with a dual coaction $\widehat{\alpha}: A \times_{\alpha} G \rightarrow$ $M\left(\left(A \times_{\alpha} G\right) \otimes C^{*}(G)\right)$ on the full crossed product $A \times_{\alpha} G$ by an action $\alpha: G \rightarrow \operatorname{Aut}(A)$, then there exists also a full version of Mansfield's theorem. More precisely, for $B=A \times_{\alpha} G$ and $\delta=\widehat{\alpha}$ the authors constructed a natural imprimitivity bimodule between the full crossed products

$$
B \times{ }_{\delta} G \times \times_{\delta \mid} N \quad \text { and } \quad B \times \times_{\delta \mid} G / N,
$$

which actually factors through the reduced version of [11] by passing to appropriate quotients. Moreover, because $\delta$ is dual to an action, the construction of the bimodule became much more natural and it even allowed versions of Mansfield's imprimitivity theorems for possibly non-normal subgroups of $G$. Note that the big advantage of working with full crossed products rather than with the spatially defined reduced ones is that full crossed products can be defined via universal properties, which, in general, are not available for the study of the reduced crossed products. 
The main purpose of this paper is to extend the results of [4] to arbitrary coactions of discrete groups. The main idea is to use the strong relationship between coactions of a discrete group $G$ and Fell bundles over $G$ as observed by the second author in [18] (see also [5]): if $\delta: A \rightarrow A \otimes C^{*}(G)$ is a coaction of the discrete group $G$ on the $C^{*}$-algebra $A$, then the set of spectral subspaces $\mathscr{A}=\left\{A_{s}: s \in G\right\}$ with $A_{s}=\{a \in A: \delta(a)=a \otimes s\}$ forms a Fell bundle over $G$. For any such bundle we can form the full and the reduced cross-sectional algebras $C^{*}(\mathscr{A})$ and $C_{r}^{*}(\mathscr{A})$ of $\mathscr{A}$, which are completions of the *-algebra $\Gamma_{c}(\mathscr{A})$ of finitely supported sections with respect to the maximal and minimal topologically graded $C^{*}$-norms (see [7]). We also have $\Gamma_{c}(\mathscr{A}) \subseteq A$ as a dense subalgebra, and the identity map on $\Gamma_{c}(\mathscr{A})$ induces quotient maps $C^{*}(\mathscr{A}) \rightarrow$ $A \rightarrow C_{r}^{*}(\mathscr{A})$. There are canonical coactions $\delta^{m}: C^{*}(\mathscr{A}) \rightarrow C^{*}(\mathscr{A}) \otimes C^{*}(G)$ and $\delta^{n}: C_{r}^{*}(\mathscr{A}) \rightarrow C_{r}^{*}(\mathscr{A}) \otimes C^{*}(G)$ determined by $a_{s} \mapsto a_{s} \otimes s$. The quotient maps $C^{*}(\mathscr{A}) \rightarrow A \rightarrow C_{r}^{*}(\mathscr{A})$ are equivariant with respect to $\delta^{m}, \delta$, and $\delta^{n}$, and it is shown in [5, Lemma 2.1] that they induce isomorphisms

$$
C^{*}(\mathscr{A}) \times_{\delta^{m}} G \stackrel{\cong}{\rightrightarrows} A \times_{\delta} G \stackrel{\cong}{\rightarrow} C_{r}^{*}(\mathscr{A}) \times_{\delta^{n}} G
$$

of the crossed products. It is actually checked in [5, Section 2] that the coaction $\delta^{n}$ on the reduced cross-sectional algebra $C_{r}^{*}(\mathscr{A})$ constructed above coincides with the normalization of $\delta$. On the other side, $\delta^{m}$ should be regarded as a maximalization of $\delta$, thus explaining our notation.

As our main result, we shall derive a new version of Mansfield's imprimitivity theorem, which works for full crossed products and arbitrary subgroups of a discrete group $G$. To be more precise, we show that for any subgroup $H$ of $G$ there exists a canonical $C^{*}(\mathscr{A}) \times_{\delta^{m}} G \times \widehat{\delta}^{m} H-C^{*}(\mathscr{A} \times$ $G / H)$ imprimitivity bimodule $X$, where $C^{*}(\mathscr{A} \times G / H)$ denotes the maximal cross-sectional algebra of the Fell bundle $\mathscr{A} \times G / H$ over the transformation groupoid $G \times G / H$. We then show that this bimodule naturally factors to a $C_{r}^{*}(\mathscr{A}) \times{\delta^{n}}^{n} G \times \widehat{\delta}^{n}, r H-C_{r}^{*}(\mathscr{A} \times G / H)$ imprimitivity bimodule for the reduced cross-sectional algebras on both sides. Note that if $H$ is normal, we have $C^{*}(\mathscr{A} \times G / H)=C^{*}(\mathscr{A}) \times \delta_{\delta^{m} \mid} G / H$ and $C_{r}^{*}(\mathscr{A} \times G / H)=C_{r}^{*}(\mathscr{A}) \times{ }_{\delta^{n} \mid} G / H$, where $\delta^{m} \mid$ and $\delta^{n} \mid$ denote the restrictions of $\delta^{m}$ and $\delta^{n}$ to $G / H$. Using the fact that an arbitrary coaction $\delta: A \rightarrow A \otimes C^{*}(G)$ of a discrete group $G$ "lies between" $\delta^{m}$ and $\delta^{n}$, we derive an "intermediate" imprimitivity theorem for $\delta$ : if $H$ is a normal subgroup of $G$ then $A \times_{\delta \mid} G / H$ is canonically Morita equivalent to a crossed product $A \times \times_{\delta} G \times \widehat{\delta}_{, \mu} H$ "lying between" the full crossed product $A \times{ }_{\delta} G \times \times_{\widehat{\delta}} H$ and the reduced crossed product $A \times{ }_{\delta} G \times{ }_{\widehat{\delta}, r} H$.

We obtain an interesting consequence concerning amenability of Fell bundles in the sense of Exel [7]: a Fell bundle $\mathscr{A}$ over $G$ is amenable if and only if its dual action $\widehat{\delta^{m}}$ of $G$ on $C^{*}(\mathscr{A}) \times_{\delta^{m}} G$ is amenable in the (weak) sense that 
the full and reduced crossed products coincide (recall that Exel defined a Fell bundle $\mathscr{A}$ to be amenable if the regular representation $C^{*}(\mathscr{A}) \rightarrow C_{r}^{*}(\mathscr{A})$ is an isomorphism). This result potentially allows us to restrict questions related to amenability of Fell bundles to the special (and better understood) case of actions. As a further application of our general imprimitivity theorem we shall derive a characterization of induced coactions as introduced in [5]: a coaction $\delta^{m}: C^{*}(\mathscr{A}) \rightarrow C^{*}(\mathscr{A}) \otimes C^{*}(G)$ is induced from a quotient group $G / H$ if and only if the dual action $\widehat{\delta}^{m}$ is twisted over $H$ in the sense of Green [9].

\section{Preliminaries}

Throughout $\S 2-\$ 4 G$ will be a discrete group. We adopt the conventions of [5], [17], [18] for coactions of groups on $C^{*}$-algebras, and of [7], [8] for Fell bundles. We will need the more general notion of Fell bundles over discrete groupoids, for which we refer to [15]. If $\mathscr{B}$ is a Fell bundle over a discrete groupoid $\mathscr{G}$, we let $\Gamma_{c}(\mathscr{B})$ denote the *-algebra of finitely supported sections. A homomorphism of $\mathscr{B}$ into a $C^{*}$-algebra $C$ is a map $\phi: \mathscr{B} \rightarrow C$ which is linear on each fiber $B_{s}$, is as multiplicative as makes sense, and preserves adjoints. A representation of $\mathscr{B}$ on a Hilbert space $\mathscr{H}$ is a homomorphism $\pi: \mathscr{B} \rightarrow \mathscr{L}(\mathscr{H})$, and we say $\pi$ is nondegenerate if $\overline{\operatorname{span}}\{\pi(\mathscr{B}) \mathscr{H}\}=\mathscr{H}$. A $C^{*}$-algebra $B$ is the enveloping $C^{*}$-algebra of a ${ }^{*}$-algebra $B_{0}$ if the supremum of the $C^{*}$-seminorms on $B_{0}$ is finite and $B$ is the Hausdorff completion of $B_{0}$ in this largest $C^{*}$-seminorm.

Proposition 2.1. If $\mathscr{B}$ is a Fell bundle over the discrete groupoid $\mathscr{G}$, then the ${ }^{*}$-algebra $\Gamma_{c}(\mathscr{B})$ has an enveloping $C^{*}$-algebra, which we denote by $C^{*}(\mathscr{B})$.

Proof. Let $\Pi$ be a representation of $\Gamma_{c}(\mathscr{B})$, and let $b=\sum_{x \in \mathscr{G}} b_{x} \in \Gamma_{c}(\mathscr{B})$, with $b_{x} \in B_{x}$ finitely nonzero. Using that $\left.\Pi\right|_{B_{d(x)}}$ is a $*$-representation of the $C^{*}$-algebra $B_{d(x)}$ we get $\left\|\Pi\left(b_{x}\right)\right\|^{2}=\left\|\Pi\left(b_{x}^{*} b_{x}\right)\right\| \leq\left\|b_{x}^{*} b_{x}\right\|=\left\|b_{x}\right\|^{2}$, which implies that $\|\Pi(b)\| \leq \sum_{x}\left\|b_{x}\right\|$. But this suffices to establish the conclusion.

COROLlaRY 2.2. The assignment $\left.\Pi \mapsto \Pi\right|_{\mathscr{B}}$ gives a one-to-one correspondence between homomorphisms of $C^{*}(\mathscr{B})$ and of $\mathscr{B}$, and between nondegenerate representations of $C^{*}(\mathscr{B})$ and of $\mathscr{B}$.

Proof. Since there is an obvious one to one correspondence between homomorphisms of $\mathscr{B}$ and $\Gamma_{c}(\mathscr{B})$, the result follows from Proposition 2.1.

Let $\alpha$ be an action of $G$ on a $C^{*}$-algebra $B$, and let $B \times G$ be the associated semidirect product Fell bundle over $G$ as in [8], so that $C^{*}(B \times G)$ is canonically isomorphic to the crossed product $B \times_{\alpha} G$. For reference, the operations on 
the bundle are

$$
(b, s)(c, t)=\left(b \alpha_{s}(c), s t\right) \text { and }(b, s)^{*}=\left(\alpha_{s^{-1}}(b)^{*}, s^{-1}\right) .
$$

Often it is convenient to restrict attention to a dense *-subalgebra $B_{0}$ of $B$, and we write $\Gamma_{c}\left(B_{0} \times G\right)$ for the linear span of $B_{0} \times G$ in $\Gamma_{c}(B \times G)$.

Lemma 2.3. Let $(B, G, \alpha)$ be an action, and suppose $B$ is the enveloping $C^{*}$-algebra of an $\alpha$-invariant ${ }^{*}$-subalgebra $B_{0}$. Then $B \times{ }_{\alpha} G$ is the enveloping $C^{*}$-algebra of $\Gamma_{c}\left(B_{0} \times G\right)$.

Proof. Proposition 2.1 implies that $B \times{ }_{\alpha} G$ is the enveloping $C^{*}$-algebra of $\Gamma_{c}(B \times G)$, so it suffices to show that every representation $\Pi$ of $B_{0} \times G$ extends to a representation of the Fell bundle $B \times G$. But using the representation $\pi$ of $B_{0}$ defined by $\pi(b)=\Pi(b, e)$ one can easily check that $\Pi$ restricts to a bounded linear map on each fiber $\left(B_{0}, s\right)$.

Now let $\delta$ be a coaction of $G$ on a $C^{*}$-algebra $A$, and let $\mathscr{A}$ be the associated Fell bundle. For any subgroup $H$ of $G$, we get a Fell bundle $\mathscr{A} \times G / H$ over the discrete transformation groupoid $G \times G / H$. For reference, the operations on $\mathscr{A} \times G / H$ are

$$
\left(a_{s}, \operatorname{tr} H\right)\left(b_{t}, r H\right)=\left(a_{s} b_{t}, r H\right) \text { and }\left(a_{s}, t H\right)^{*}=\left(a_{s}^{*}, s t H\right) .
$$

We define a notion of covariant representation for the "restricted coaction" $(A, G / H, \delta \mid$ ) (whatever that might be), and show in Proposition 2.7 that $C^{*}(\mathscr{A} \times G / H)$ is characterized by a universal property for covariant representations. In Corollary 2.8 we deduce that $C^{*}(\mathscr{A} \times G / H)$ reduces to the usual crossed product $A \times{ }_{\delta \mid} G / H$ when $H$ is normal and $\delta$ is maximal (that is, $\left.A=C^{*}(\mathscr{A})\right)$. Recall that the restriction $\delta \mid$ of a coaction $\delta: A \rightarrow A \otimes C^{*}(G)$ to the quotient group $G / H$ is defined by

$$
\delta \mid=\left(\mathrm{id}_{A} \otimes q\right) \circ \delta: A \rightarrow A \otimes C^{*}(G / H),
$$

where $q: C^{*}(G) \rightarrow C^{*}(G / H)$ denotes the quotient map. Proposition 2.7 also shows that when $H$ is non-normal $C^{*}(\mathscr{A} \times G / H)$ is analogous to the "full crossed product by the (dual) coaction of the homogeneous space $G / H$ " in [4, Section 2].

Definition 2.4. A covariant representation of $(A, G / H, \delta \mid)$ is a pair $(\pi, \mu)$, where $\pi$ is a nondegenerate representation of $A$ and $\mu$ is a nondegenerate representation of $c_{0}(G / H)$ on the same Hilbert space, such that

$$
\pi\left(a_{s}\right) \mu\left(\chi_{t H}\right)=\mu\left(\chi_{s t H}\right) \pi\left(a_{s}\right) \quad \text { for all } s, t \in G, a_{s} \in A_{s},
$$


where $\chi_{t H}$ denotes the characteristic function of the singleton subset $\{t H\}$ of $G / H$.

Remark 2.5. When $H$ is normal, if follows from [18, Lemma 2.2] that the above definition is equivalent to the more usual definition of covariant representation of the coaction $(A, G / H, \delta \mid)$, namely

$$
(\pi \otimes \mathrm{id}) \circ \delta(a)=\operatorname{Ad} \mu \otimes \operatorname{id}\left(w_{G / H}\right)(\pi(a) \otimes 1) \quad \text { for } a \in A,
$$

where $w_{G / H}$ is the unitary element of $M\left(c_{0}(G / H) \otimes C^{*}(G / H)\right)=$ $c_{b}\left(G / H, C^{*}(G / H)\right)$ defined by $w_{G / H}(s H)=s H$, where on the right hand side we view $s H$ as an element of $C^{*}(G / H)$. However, when $H$ is non-normal there is no such object $w_{G / H}$.

Proposition 2.6. Let $(\pi, \mu)$ be a covariant representation of $(A, G, \delta)$, and let $\mu \mid$ denote the restriction $\left.\mu\right|_{c_{0}(G / H)}$. Then $(\pi, \mu \mid)$ is a covariant representation of $(A, G / H, \delta \mid)$.

Proof. We need only verify the covariance condition (2.1). Take $s, t \in G$ and $a_{s} \in A_{s}$. Since $\chi_{t H}=\sum_{h \in H} \chi_{t h}$, the sum converging in the strict topology of $c_{b}(G)=M\left(c_{0}(G)\right)$, we have

$$
\begin{aligned}
\pi\left(a_{s}\right) \mu \mid\left(\chi_{t H}\right) & =\pi\left(a_{s}\right) \sum_{h} \mu\left(\chi_{t h}\right)=\sum_{h} \pi\left(a_{s}\right) \mu\left(\chi_{t h}\right)=\sum_{h} \mu\left(\chi_{s t h}\right) \pi\left(a_{s}\right) \\
& =\left(\sum_{h} \mu\left(\chi_{s t h}\right)\right) \pi\left(a_{s}\right)=\mu \mid\left(\chi_{s t H}\right) \pi\left(a_{s}\right) .
\end{aligned}
$$

Proposition 2.7. For every covariant representation $(\pi, \mu)$ of $(A, G / H, \delta \mid)$, there exists a unique nondegenerate representation $\pi \times \mu$ of $C^{*}(\mathscr{A} \times G / H)$ such that

$$
\pi \times \mu(a, t H)=\pi(a) \mu\left(\chi_{t H}\right) \quad \text { for all } a \in \mathscr{A}, t \in G .
$$

Moreover, in the case $A=C^{*}(\mathscr{A})$, every nondegenerate representation of $C^{*}(\mathscr{A} \times G / H)$ arises this way.

Proof. First assume $(\pi, \mu)$ is a covariant representation of $(A, G / H, \delta \mid)$. It is easy to check that $(a, t H) \mapsto \pi(a) \mu\left(\chi_{t H}\right)$ is a representation of the Fell bundle $\mathscr{A} \times G / H$, and moreover this representation is nondegenerate since both $\pi$ and $\mu$ are. So, by Corollary 2.2 there exists a unique representation $\pi \times \mu$ of $C^{*}(\mathscr{A} \times G / H)$ satisfying the compatibility condition (2.2).

Conversely, assume $A=C^{*}(\mathscr{A})$, and let $\Pi$ be a nondegenerate representation of $C^{*}(\mathscr{A} \times G / H)$. For each coset $t H \in G / H$ define a representation 
$\sigma_{t H}$ of the unit fiber algebra $A_{e}$ by

$$
\sigma_{t H}\left(a_{e}\right)=\Pi\left(a_{e}, t H\right) .
$$

Fix a bounded approximate identity $\left\{d_{i}\right\}$ for $A_{e}$, and for each $t H \in G / H$ put

$$
p_{t H}=\lim \sigma_{t H}\left(d_{i}\right),
$$

the limit taken in the weak operator topology. Then $\left\{p_{t H}: t H \in G / H\right\}$ is an orthogonal family of projections, hence determines a representation $\mu$ of $c_{0}(G / H)$ such that $\mu\left(\chi_{t H}\right)=p_{t H}$. Computations similar to those in the proof of [5, Theorem 3.3] show that for all $a \in \mathscr{A}$ the sum

$$
\pi(a):=\sum_{t H \in G / H} \Pi(a, t H)
$$

converges in the weak operator topology and determines a representation $\pi$ of $A$, and moreover $(\pi, \mu)$ is a covariant representation of $(A, G / H, \delta \mid)$ such that $\Pi=\pi \times \mu$.

Notice that if $A \neq C^{*}(\mathscr{A})$, the case $H=G$ shows that there might be strictly fewer covariant representations of $(A, G / H, \delta \mid)$ than representations of $C^{*}(\mathscr{A} \times G / H)$. From Proposition 2.7 and the universal properties of crossed products by coactions with respect to covariant representations we get

COROLlary 2.8. If $H$ is normal then there is a unique isomorphism $C^{*}(\mathscr{A}) \times \delta_{\delta^{m} \mid} G / H \cong C^{*}(\mathscr{A} \times G / H)$ mapping $j_{C^{*}(\mathscr{A})}\left(a_{s}\right) j_{G / H}\left(\chi_{t H}\right)$ to $\left(a_{s}, t H\right)$ (where $j_{\left.C^{*} \mathscr{A}\right)}$ and $j_{G / H}$ denote the canonical maps of $C^{*}(\mathscr{A})$ and $c_{0}(G / H)$ into $M\left(C^{*}(\mathscr{A}) \times \delta^{m} \mid G / H\right)$ ).

Now we recall from [15] Kumjian's construction of what we call the reduced cross-sectional algebra of a Fell bundle $\mathscr{B}$ over a groupoid $\mathscr{G}$. Kumjian only needed $\mathscr{G}$ to be $r$-discrete, but our $\mathscr{G}$ will actually be discrete. $\Gamma_{c}(\mathscr{B})$ is given a pre-Hilbert $\Gamma_{c}\left(\mathscr{B}^{0}\right)$-module structure (where $\mathscr{B}^{0}$ is the restricted bundle $\left.\mathscr{B}\right|_{\mathscr{G} 0}$ over the unit space $\mathscr{G}^{0}$ ), with inner product

$$
\langle f, g\rangle_{\Gamma_{c}\left(\mathscr{B}^{0}\right)}=\left.\left(f^{*} g\right)\right|_{\mathscr{G} 0} \quad \text { for } f, g \in \Gamma_{c}(\mathscr{B}) .
$$

Here $\Gamma_{c}\left(\mathscr{B}^{0}\right)$ is regarded as a subalgebra of $\Gamma_{0}\left(\mathscr{B}^{0}\right)$, the $C_{0}$-section algebra of the $C^{*}$-bundle $\mathscr{B}^{0}$. Then the completion $L^{2}(\mathscr{B})$ of $\Gamma_{c}(\mathscr{B})$ is a full Hilbert $\Gamma_{0}\left(\mathscr{B}^{0}\right)$-module. Left multiplication in $\Gamma_{c}(\mathscr{B})$ extends to a nondegenerate action of $\Gamma_{c}(\mathscr{B})$ on $L^{2}(\mathscr{B})$ by adjointable operators, and the completion of $\Gamma_{c}(\mathscr{B})$ in the norm of this representation is the reduced cross-sectional algebra $C_{r}^{*}(\mathscr{B})$. The identity map on $\mathscr{B}$ extends uniquely to a surjective homomorphism, which we call the regular representation, of $C^{*}(\mathscr{B})$ onto $C_{r}^{*}(\mathscr{B})$. Further, 
we call a Fell bundle $\mathscr{B}$ over a discrete groupoid $\mathscr{G}$ amenable if the regular representation of $C^{*}(\mathscr{B})$ onto $C_{r}^{*}(\mathscr{B})$ is faithful. Note that in the special case where $\mathscr{G}$ is actually a group, this is the same terminology used by Exel [7]. Note also that in the case of an action $(B, G, \alpha), C_{r}^{*}(B \times G)$ can be identified with the reduced crossed product $B \times_{\alpha, r} G$.

Consider the case $\mathscr{B}=\mathscr{A} \times G / H$, where $\mathscr{A}$ is a Fell bundle over $G$, and $\mathscr{G}=G \times G / H$. The unit space $\mathscr{G}^{0}=\{e\} \times G / H$ can be identified with $G / H$. Then $\mathscr{B}^{0}=A_{e} \times G / H$, a trivial $C^{*}$-bundle, so the $C_{0}$-section algebra is

$$
\Gamma_{0}\left(A_{e} \times G / H\right)=A_{e} \otimes c_{0}(G / H)=c_{0}\left(G / H, A_{e}\right) .
$$

The inner product on $\Gamma_{c}(\mathscr{B})=\Gamma_{c}(\mathscr{A} \times G / H)$ is

$$
\langle f, g\rangle_{A_{e} \otimes c_{0}(G / H)}=\left.\left(f^{*} g\right)\right|_{\{e\} \times G / H} .
$$

Writing $f$ as a finitely nonzero sum $\sum_{s, t H}\left(f_{s, t H}, t H\right)$, and similarly for $g$, we arrive at

$$
\begin{aligned}
\left\langle\sum_{s, t H}\left(f_{s, t H}, t H\right), \sum_{u, v H}\left(g_{u, v H}, v H\right)\right\rangle_{A_{e} \otimes c_{0}(G / H)} & \\
& =\sum_{s, t H}\left(f_{s, t H}^{*} g_{s, t H}, t H\right) \\
& =\sum_{t H \in G / H}\left(\sum_{s \in G}\left\langle f_{s, t H}, g_{s, t H}\right\rangle_{A_{e}}, t H\right) .
\end{aligned}
$$

In particular, for generators $\left(a_{s}, t H\right),\left(b_{u}, v H\right) \in \mathscr{A} \times G / H$ we have $\left\langle\left(a_{s}, t H\right),\left(b_{u}, v H\right)\right\rangle_{A_{e} \otimes c_{0}(G / H)}=\left(a_{s}^{*} b_{u}, t H\right)$ if $s=u, t H=v H$ (and 0 else).

The regular representation, which we denote by $\Lambda$, of the cross-sectional algebra $C^{*}(\mathscr{A} \times G / H)$ on the Hilbert $A_{e} \otimes c_{0}(G / H)$-module $L^{2}(\mathscr{A} \times G / H)$ is given by

$$
\Lambda\left(a_{s}, t H\right)\left(b_{u} \otimes \chi_{v H}\right)=a_{s} b_{u} \otimes \chi_{v H} \quad \text { if } t H=v H \text { (and } 0 \text { else), }
$$

and $C_{r}^{*}(\mathscr{A} \times G / H)$ is the image of $C^{*}(\mathscr{A} \times G / H)$ in $\mathscr{L}\left(L^{2}(\mathscr{A} \times G / H)\right)$.

We want to identify the reduced cross-sectional algebra $C_{r}^{*}(\mathscr{A} \times G / H)$ with the image $\operatorname{Im} j_{C^{*}(\mathscr{A})} \times j_{G} \mid$ of $C^{*}(\mathscr{A} \times G / H)$ in $M\left(C^{*}(\mathscr{A}) \times{ }_{\delta^{m}} G\right)$, where $j_{C^{*}(\mathscr{A})}$ and $j_{G}$ denote the canonical maps of $C^{*}(\mathscr{A})$ and $c_{0}(G)$ into $M\left(C^{*}(\mathscr{A}) \times \delta^{m} G\right)$ and $j_{C^{*}(\mathscr{A})} \times j_{G} \mid$ is the corresponding $*$-homomorphism of $C^{*}(\mathscr{A} \times G / H)$ as described in Proposition 2.7. For this we need the following technical lemma on Hilbert modules, which is an easy modification of [21, Lemma 2.5]. The 
essential idea is that a linear map between Hilbert modules which preserves inner products is automatically a module homomorphism. Let $C$ and $D$ be $C^{*}$ algebras, let $Z$ be a (right) Hilbert $D$-module, and suppose $C$ is represented by adjointable operators on $Z$. If $Z$ is full as a Hilbert $D$-module and the action of $C$ on $Z$ is nondegenerate, we say $Z$ is a right-Hilbert $C-D$ bimodule. We use the notation ${ }_{C} Z_{D}$ to indicate that the coefficient algebras of the bimodule $Z$ are $C$ and $D$. If $C_{0}$ and $D_{0}$ are dense ${ }^{*}$-subalgebras and $Z_{0}$ is a dense linear subspace such that $C_{0} Z_{0} \cup Z_{0} D_{0} \subseteq Z_{0}$ and $\left\langle Z_{0}, Z_{0}\right\rangle_{D} \subseteq D_{0}$, we say ${ }_{C} Z_{D}$ is the completion of the right-pre-Hilbert bimodule $C_{0}\left(Z_{0}\right)_{D_{0}}$.

Lemma 2.9 (cf. [21, Lemma 2.5]). Suppose ${ }_{C} Z_{D}$ and ${ }_{E} W_{F}$ are right-Hilbert bimodules such that ${ }_{C} Z_{D}$ is the completion of a right-pre-Hilbert bimodule $C_{0}\left(Z_{0}\right)_{D_{0}}$, and suppose we are given homomorphisms $\phi: C \rightarrow E$ and $\psi: D \rightarrow$ $F$ and a linear map $\Psi: Z_{0} \rightarrow W$ with dense range such that for all $c \in C_{0}$ and $z, w \in Z_{0}$ we have

(i) $\Psi(c z)=\phi(c) \Psi(z)$, and

(ii) $\langle\Psi(z), \Psi(w)\rangle_{F}=\psi\left(\langle z, w\rangle_{D}\right)$.

Then $\Psi$ extends uniquely to a right-Hilbert bimodule homomorphism of ${ }_{C} Z_{D}$ onto ${ }_{E} W_{F}$. Moreover, if $Z$ and $W$ are actually imprimitivity bimodules, this extension of $\Psi$ is an imprimitivity bimodule homomorphism.

Proposition 2.10. With the notation preceding Lemma 2.9, the two homomorphisms $\Lambda$ and $j_{C^{*}(\mathscr{A})} \times j_{G} \mid$ of $C^{*}(\mathscr{A} \times G / H)$ have the same kernel.

Proof. It suffices to produce isomorphic right-Hilbert $C^{*}(\mathscr{A} \times G / H)-A_{e}$ bimodules $Y$ and $Z$ such that the homomorphisms of $C^{*}(\mathscr{A} \times G / H)$ into $\mathscr{L}(Y)$ and $\mathscr{L}(Z)$ have the same kernels as $\Lambda$ and $j_{C^{*}(\mathscr{A})} \times j_{G} \mid$, respectively. To get $Y$, note that $A_{e} \otimes c_{0}(G / H)$ acts faithfully by adjointable operators on the external tensor product $A_{e} \otimes \ell^{2}(G)$ of the Hilbert $A_{e}$-module $A_{e}$ and the Hilbert space $\ell^{2}(G)$, via

$$
(a \otimes f)(b \otimes g)=a b \otimes f g \quad \text { for } a, b \in A_{e}, f \in c_{0}(G / H), g \in \ell^{2}(G) .
$$

Thus, the associated homomorphism of $C^{*}(\mathscr{A} \times G / H)$ into the adjointable operators on the balanced tensor product $L^{2}(\mathscr{A} \times G / H) \otimes_{A_{e} \otimes c_{0}(G / H)}\left(A_{e} \otimes\right.$ $\left.\ell^{2}(G)\right)$ has the same kernel as $\Lambda$.

In order to construct the bimodule $Z$, first note that, since the coaction $\delta^{n}$ on $C_{r}^{*}(\mathscr{A})$ is the normalization of $\delta^{m}$, the crossed product $C^{*}(\mathscr{A}) \times \delta^{m} G$ has a faithful representation on the Hilbert $A_{e}$-module $L^{2}(\mathscr{A}) \otimes \ell^{2}(G)$. The action is given on the generators by

$$
\left(a_{s}, t\right)\left(b_{u} \otimes \chi_{v}\right)=a_{s} b_{u} \otimes \lambda_{s} M_{\chi_{t}} \chi_{v}=a_{s} b_{u} \otimes \chi_{s v} \quad \text { if } t=v \text { (and } 0 \text { else). }
$$


If we compose this representation with $j_{C^{*}(\mathscr{A})} \times j_{G} \mid: C^{*}(\mathscr{A} \times G / H) \rightarrow$ $M\left(C^{*}(\mathscr{A}) \times \delta^{m} G\right), C^{*}(\mathscr{A} \times G / H)$ acts on $L^{2}(\mathscr{A}) \times \ell^{2}(G)$ by

$$
\left(a_{s}, t H\right)\left(b_{u} \otimes \chi_{v}\right)=a_{s} b_{u} \otimes \chi_{s v} \quad \text { if } v \in t H \text { (and } 0 \text { else). }
$$

Thus for $Z$ we take $L^{2}(\mathscr{A}) \otimes \ell^{2}(G)$ equipped with this action.

We want to define an isomorphism $\Psi$ between the Hilbert $A_{e}$-modules $L^{2}(\mathscr{A} \times G / H) \otimes_{A_{e} \otimes c_{0}(G / H)}\left(A_{e} \otimes \ell^{2}(G)\right)$ and $L^{2}(\mathscr{A}) \otimes \ell^{2}(G)$. We begin by defining $\Psi$ on the generators:

$$
\Psi\left(\left(a_{s}, t H\right) \otimes\left(b \otimes \chi_{r}\right)\right)=a_{s} b \otimes \chi_{s r} \quad \text { if } r \in t H \text { (and } 0 \text { else). }
$$

and then extending additively. This gives a linear map from $\Gamma_{c}(\mathscr{A} \times G / H) \odot$ $\left(A_{e} \odot C_{c}(G)\right)$ to $L^{2}(\mathscr{A}) \otimes \ell^{2}(G)$ with dense range. We show $\Psi$ preserves inner products. For $a_{s}, b_{u} \in \mathscr{A}, t H, v H \in G / H, c, d \in A_{e}$, and $r, w \in G$ we have

$$
\begin{aligned}
& \left\langle\Psi\left(\left(a_{s}, t H\right) \otimes\left(c \otimes \chi_{r}\right)\right), \Psi\left(\left(b_{u}, v H\right) \otimes\left(d \otimes \chi_{w}\right)\right)\right\rangle_{A_{e}} \\
& \quad=\left\langle a_{s} c \otimes \chi_{s r}, b_{u} d \otimes \chi_{u w}\right\rangle_{A_{e}} \quad \text { if } r \in t H, w \in v H \text { (and } 0 \text { else) } \\
& \quad=\left\langle a_{s} c, b_{u} d\right\rangle_{A_{e}}\left\langle\chi_{s r}, \chi_{u w}\right\rangle \quad \text { if } r \in t H, w \in v H \text { (and } 0 \text { else) } \\
& \quad=c^{*} a_{s}^{*} b_{u} d \quad \text { if } s=u, s r=u w, r \in t H, w \in v H \text { (and } 0 \text { else). }
\end{aligned}
$$

On the other hand,

$$
\begin{aligned}
& \left\langle\left(a_{s}, t H\right) \otimes\left(c \otimes \chi_{r}\right),\left(b_{u}, v H\right) \otimes\left(d \otimes \chi_{w}\right)\right\rangle_{A_{e}} \\
& \quad=\left\langle c \otimes \chi_{r},\left\langle\left(a_{s}, t H\right),\left(b_{u}, v H\right)\right\rangle_{A_{e} \otimes c_{0}(G / H)}\left(d \otimes \chi_{w}\right)\right\rangle_{A_{e}} \\
& \quad=\left\langle c \otimes \chi_{r},\left(a_{s}^{*} b_{u}, t H\right)\left(d \otimes \chi_{w}\right)\right\rangle_{A_{e}} \quad \text { if } s=u, t H=v H \text { (and } 0 \text { else) } \\
& \quad=\left\langle c \otimes \chi_{r}, a_{s}^{*} b_{u} d \otimes \chi_{w}\right\rangle_{A_{e}} \quad \text { if } s=u, t H=v H, w \in t H \text { (and } 0 \text { else) } \\
& \quad=c^{*} a_{s}^{*} b_{u} d\left\langle\chi_{r}, \chi_{w}\right\rangle \quad \text { if } s=u, t H=v H, w \in t H \text { (and } 0 \text { else) } \\
& \quad=c^{*} a_{s}^{*} b_{u} d \quad \text { if } s=u, t H=v H, w \in t H, r=w \text { (and } 0 \text { else). }
\end{aligned}
$$

Since the two sets of conditions

$\{s=u, s r=u w, r \in t H, w \in v H\}$ and $\{s=u, t H=v H, w \in t H, r=w\}$

are equivalent, $\Psi$ preserves the inner products.

A similar computation shows that $\Psi$ preserves the left module actions. Thus, by Lemma $2.9, \Psi$ extends to a surjective right-Hilbert bimodule homomorphism, which we still denote by $\Psi$. Since the right hand coefficient homomorphism is the identity map on $A_{e}, \Psi$ is actually an isomorphism.

REMARK 2.11. When $H=\{e\}$ the result shows that the reduced crosssectional algebra $C_{r}^{*}(\mathscr{A} \times G)$ of the Fell bundle $\mathscr{A} \times G$ over $G \times G$ is isomorphic to the crossed product $C^{*}(\mathscr{A}) \times{ }_{\delta^{m}} G$. Hence, the Fell bundle $\mathscr{A} \times G$ is 
always amenable in the sense that the regular representation $\Lambda: C^{*}(\mathscr{A} \times G) \rightarrow$ $C_{r}^{*}(\mathscr{A} \times G)$ is faithful.

COROLlaRY 2.12. If $H$ is normal then there is a unique isomorphism $C_{r}^{*}(\mathscr{A}) \times_{\delta^{n} \mid} G / H \cong C_{r}^{*}(\mathscr{A} \times G / H)$ mapping $j_{C_{r}^{*}(\mathscr{A})}\left(a_{s}\right) j_{G / H}\left(\chi_{t H}\right)$ to $\left(a_{s}, t H\right)$.

Proof. We must show that $j_{C_{r}^{*}(\mathscr{A})} \times j_{G / H}$ has the same kernel as the regular representation $\Lambda: C^{*}(\mathscr{A} \times G / H) \rightarrow C_{r}^{*}(\mathscr{A} \times G / H)$. By Proposition 2.10, it suffices to show that the homomorphisms $j_{C_{r}^{*}(\mathscr{A})} \times j_{G / H}$ and $j_{C^{*}(\mathscr{A})} \times j_{G} \mid$ of $C^{*}(\mathscr{A} \times G / H)$ have the same kernel. These maps fit into a commutative diagram

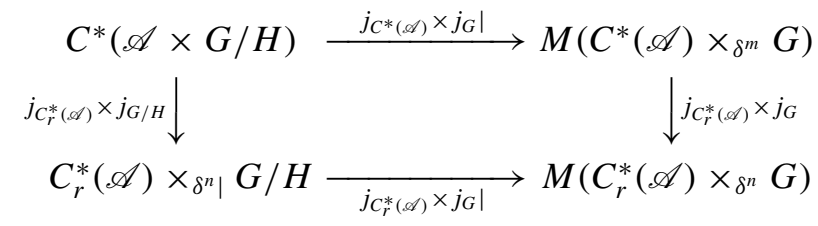

The right hand map is an isomorphism. Since the coaction $\delta^{n}$ is normal, by [11, Lemma 3.1] the bottom map is injective. The result follows.

\section{The imprimitivity theorem}

In this section we prove the imprimitivity theorems for coactions of discrete groups. Starting with a Fell bundle $\mathscr{A}$ over $G$, we first construct a $C^{*}(\mathscr{A}) \times_{\delta^{m}}$ $G \times \widehat{\delta^{m}} H-C^{*}(\mathscr{A} \times G / H)$ imprimitivity bimodule $X$, where $\delta^{m}$ is the canonical coaction of $G$ on $C^{*}(\mathscr{A})$. As usual, we work with dense subspaces. For $C^{*}(\mathscr{A} \times$ $G / H)$ we take the dense ${ }^{*}$-subalgebra $C_{0}:=\Gamma_{c}(\mathscr{A} \times G / H)$, where we remind the reader to regard $\mathscr{A} \times G / H$ as a Fell bundle over the groupoid $G \times G / H$. For $C^{*}(\mathscr{A}) \times_{\delta^{m}} G \times \widehat{\delta^{m}} H$ we form the corresponding dense *-subalgebra $B_{0}:=$ $\Gamma_{c}(\mathscr{A} \times G \times H)$, and we put $X_{0}=\Gamma_{c}(\mathscr{A} \times G)$. For reference, the operations on the *-algebra $B_{0}$ are given on the generators by

$$
\begin{aligned}
\left(a_{s}, t, h\right)\left(a_{u}, v, l\right) & =\left(a_{s} a_{u}, v h^{-1}, h l\right) \quad \text { if } t h=u v \text { (and } 0 \text { else) } \\
\left(a_{s}, t, h\right)^{*} & =\left(a_{s}^{*}, s t h, h^{-1}\right) .
\end{aligned}
$$

The $B_{0}-C_{0}$ pre-imprimitivity bimodule structure on $X_{0}$ will be given on the generators by

$$
\begin{aligned}
\left(a_{s}, t\right) \cdot\left(a_{u}, v H\right) & =\left(a_{s} a_{u}, u^{-1} t\right) \quad \text { if } t H=u v H \text { (and } 0 \text { else) } \\
\left(a_{q}, r, h\right) \cdot\left(a_{s}, t\right) & =\left(a_{q} a_{s}, t h^{-1}\right) \quad \text { if } r h=s t \text { (and } 0 \text { else) } \\
\left\langle\left(a_{s}, t\right),\left(a_{u}, v\right)\right\rangle_{C_{0}} & =\left(a_{s}^{*} a_{u}, v H\right) \quad \text { if } s t=u v \text { (and } 0 \text { else) } \\
B_{0}\left\langle\left(a_{s}, t\right),\left(a_{u}, v\right)\right\rangle & =\left(a_{s} a_{u}^{*}, u t, t^{-1} v\right) \quad \text { if } t H=v H \text { (and } 0 \text { else). }
\end{aligned}
$$


TheOREm 3.1. Suppose that $\mathscr{A}$ is a Fell bundle over the discrete group $G$ and $H$ is a subgroup of $G$. The above operations make $X_{0}$ into a $B_{0}-C_{0}$ preimprimitivity bimodule. Consequently, its completion is a $C^{*}(\mathscr{A}) \times \delta^{m} G \times \widehat{\delta^{m}}$ $H-C^{*}(\mathscr{A} \times G / H)$ imprimitivity bimodule $X$.

Proof. We closely follow the lines of the proof of [5, Theorem 4.1], where we proved an imprimitivity theorem for crossed products by induced coactions. We have to check the following items:

(i) $X_{0}$ is a $B_{0}-C_{0}$ bimodule;

(ii) ${ }_{B_{0}}\langle b \cdot x, y\rangle=b_{B_{0}}\langle x, y\rangle$ and $\langle x, y \cdot c\rangle_{C_{0}}=\langle x, y\rangle_{C_{0}} c$;

(iii) $B_{0}\langle x, y\rangle^{*}={ }_{B_{0}}\langle y, x\rangle$ and $\langle x, y\rangle_{C_{0}}^{*}=\langle y, x\rangle_{C_{0}}$;

(iv) $B_{B_{0}}\langle x, y\rangle$ is linear in $x$ and $\langle x, y\rangle_{C_{0}}$ is linear in $y$;

(v) $x \cdot\langle y, z\rangle_{C_{0}}={ }_{B_{0}}\langle x, y\rangle \cdot z$;

(vi) $\operatorname{span}_{B_{0}}\left\langle X_{0}, X_{0}\right\rangle$ is dense in $B_{0}$ and $\operatorname{span}\left\langle X_{0}, X_{0}\right\rangle_{C_{0}}$ is dense in $C_{0}$;

(vii) $B_{0}\langle x, x\rangle \geq 0$ and $\langle x, x\rangle_{C_{0}} \geq 0$;

(viii) $\langle b \cdot x, b \cdot x\rangle_{C_{0}} \leq\|b\|^{2}\langle x, x\rangle_{C_{0}}$ and ${ }_{B_{0}}\langle x \cdot c, x \cdot c\rangle \leq\|c\|^{2}{ }_{B_{0}}\langle x, x\rangle$.

The verifications of the algebraic properties (i)-(v) are routine and we omit the details on this. As in [5], we prove (vi)-(vii) in one whack using Rieffel's trick: it suffices to produce nets in both $B_{0}$ and $C_{0}$, each term of which is a finite sum of the form $\sum\left\langle x_{i}, x_{i}\right\rangle$, which are approximate identities for both the algebras and the module multiplications in the inductive limit topologies.

To construct an approximate identity for $C_{0}$ let $\left\{a_{i}\right\}_{i \in I}$ be a bounded and positive approximate identity for the unit fiber $A_{e}$, and let $\mathscr{F}$ denote the family of finite subsets of $G / H$, directed by inclusion. For each $F \in \mathscr{F}$ we choose $S_{F} \subseteq G$ comprising exactly one element from each coset in $F$. Then

$$
\sum_{t \in S_{F}}\left\langle\left(a_{i}^{1 / 2}, t\right),\left(a_{i}^{1 / 2}, t\right)\right\rangle_{C_{0}}=\sum_{t H \in F}\left(a_{i}, t H\right)
$$

for all $(i, F) \in I \times \mathscr{F}$, which implies

$$
\left\{\sum_{t \in S_{F}}\left\langle\left(a_{i}^{1 / 2}, t\right),\left(a_{i}^{1 / 2}, t\right)\right\rangle_{C_{0}}\right\}_{(i, F) \in I \times \mathscr{F}}
$$

is an approximate identity (in the inductive limit topologies) for both the algebra $C_{0}$ and the right module multiplication of $C_{0}$ on $X_{0}$. For $B_{0}$, we let $\tilde{F}$ denote the finite subsets of $G$. Then the desired approximate identity in $B_{0}$ is given by the elements

$$
\sum_{t \in F} B_{0}\left|\left(a_{i}^{1 / 2}, t\right),\left(a_{i}^{1 / 2}, t\right)\right\rangle=\sum_{t \in F}\left(a_{i}, t, e\right),
$$


where $(i, F)$ runs through the directed set $I \times \tilde{\mathscr{F}}$.

In order to show (viii) we first observe that each generator $\left(a_{s}, t, h\right) \in B_{0}$ determines an adjointable operator on the pre-Hilbert $C_{0}$-module $X_{0}$ with adjoint given by the action of $\left(a_{s}, t, h\right)^{*}=\left(a_{s}^{*}, s t h, h^{-1}\right)$. The product $\left(a_{s}, t, h\right)^{*}$ $\left(a_{s}, t, h\right)=\left(a_{s}^{*} a_{s}, t, e\right)$ is a positive element of the $C^{*}$-algebra $\left(A_{e}, t, e\right)$. Extend the action of $\left(A_{e}, t, e\right)$ on $X_{0}$ to its unitization $\left(\tilde{A}_{e}, t, e\right)$ in the obvious way. We find elements $b_{e} \in \tilde{A}_{e}$ such that $\left(b_{e}^{*} b_{e}, t, e\right)=\left\|\left(a_{s}, t, h\right)\right\|^{2} 1-\left(a_{s}^{*} a_{s}, t, e\right)$. Hence, using (vii), we get

$$
\begin{aligned}
\left\|\left(a_{s}, t, h\right)\right\|^{2}\langle x, x\rangle_{C_{0}}-\left\langle\left(a_{s}, t, h\right) \cdot x\right. & \left.,\left(a_{s}, t, h\right) \cdot x\right\rangle_{C_{0}} \\
& =\left\langle\left(b_{e}, t, e\right) \cdot x,\left(b_{e}, t, e\right) \cdot x\right\rangle_{C_{0}} \geq 0 .
\end{aligned}
$$

Thus the action of $\left(a_{s}, t, h\right)$ extends to an adjointable operator on the Hilbert $C^{*}(\mathscr{A} \times G / H)$-module completion $X$, which by (i) gives us a *-homomorphism of $B_{0}$ into the $C^{*}$-algebra of adjointable module maps on $X$. By Lemma 2.3 this homomorphism extends to the enveloping $C^{*}$-algebra $C^{*}(\mathscr{A}) \times_{\delta^{m}} G \times \widehat{\delta^{m}} H$, hence must be contractive, and we arrive at the desired inequality $\langle b \cdot x, b$. $x\rangle_{C_{0}} \leq\|b\|^{2}\langle x, x\rangle_{C_{0}}$ for $b \in B_{0}$. The inequality ${ }_{B_{0}}\langle x \cdot c, x \cdot c\rangle \leq\|c\|_{B_{0}}^{2}\langle x, x\rangle$ is proved similarly.

REMARK 3.2. Theorem 3.1 can be made equivariant for appropriate coactions of $G$ on $C^{*}(\mathscr{A}) \times_{\delta^{m}} G \times \widehat{\delta^{m}} H$ and $C^{*}(\mathscr{A} \times G / H)$. The "appropriate" coaction of $G$ on $C^{*}(\mathscr{A}) \times{ }_{\delta^{m}} G \times \times_{\widehat{\delta^{m}}} H$ is $\operatorname{Inf}\left(\widehat{\delta^{m}} \mid H\right)^{\widehat{y}}$, the inflation from $H$ to $G$ of the dual coaction of the restricted action $\widehat{\delta^{m}} \mid H$. Recall that if $\epsilon: B \rightarrow B \otimes$ $C^{*}(H)$ is a coaction of $H$, then $\operatorname{Inf} \epsilon=\left(\operatorname{id} \otimes C_{H}\right) \circ \epsilon: B \rightarrow B \otimes C^{*}(G)$, where $C_{H}: C^{*}(H) \rightarrow C^{*}(G)$ denotes the natural inclusion. The "appropriate" coaction of $G$ on $C^{*}(\mathscr{A} \times G / H)$ is the decomposition coaction $\delta^{\mathrm{dec}}$, given on the generators by

$$
\delta^{\operatorname{dec}}\left(a_{s}, t H\right)=\left(a_{s}, t H\right) \otimes s .
$$

This is readily verified to give a homomorphism of the Fell bundle $\mathscr{A} \times G / H$, hence of the $C^{*}$-algebra $C^{*}(\mathscr{A} \times G / H)$, into the $C^{*}$-algebra $C^{*}(\mathscr{A} \times G / H) \otimes$ $C^{*}(G)$. Moreover it is easy to check that this homomorphism is nondegenerate and satisfies the coaction identity, and is injective because (id $\left.\otimes 1_{G}\right) \circ \delta^{\text {dec }}$ is the identity map on $C^{*}(\mathscr{A} \times G / H)$, where $1_{G}$ denotes the trivial one-dimensional representation of $G$.

Recall that $X$ is the completion of the $B_{0}-C_{0}$ pre-imprimitivity bimodule $X_{0}$ with operations given by (3.2). Adapting from [6] to our context (with nonnormal subgroups, full coactions, full crossed products, and discrete groups), we find a unique homomorphism $\delta_{X}$ of $X_{0}$ into the $\left(B_{0} \odot C^{*}(G)\right)-\left(C_{0} \odot\right.$ $\left.C^{*}(G)\right)$ pre-imprimitivity bimodule $X_{0} \odot C^{*}(G)$ such that

$$
\delta_{X}(a, t)=(a, t) \otimes t^{-1} .
$$


Hence $\delta_{X}$ extends uniquely to a homomorphism of the $C^{*}(\mathscr{A}) \times_{\delta^{m}} G \times \widehat{\delta^{m}} H-$ $C^{*}(\mathscr{A} \times G / H)$ imprimitivity bimodule $X$ into the $\left(\left(C^{*}(\mathscr{A}) \times{ }_{\delta^{m}} G \times \widehat{\delta}^{m} H\right) \otimes\right.$ $\left.G^{*}(G)\right)-\left(C^{*}(\mathscr{A} \times G / H) \otimes G^{*}(G)\right)$ imprimitivity bimodule $X \otimes C^{*}(G)$. Moreover, it is easy to verify on the generators that this homomorphism is nondegenerate and satisfies the coaction identity. Therefore $\delta_{X}$ implements a Morita equivalence between the coactions $\operatorname{Inf}\left(\widehat{\delta^{m}} \mid H\right)^{\widehat{ }}$ and $\delta^{\mathrm{dec}}$. Observe that in the extreme case $H=G$ we obtain a Morita equivalence between $\widehat{\delta^{m}}$ and $\delta^{m}$, where $\widehat{\delta^{m}}$ is the dual coaction on the maximal crossed product $C^{*}(\mathscr{A}) \times \delta^{m} G \times \widehat{\delta^{m}} G$. Note that this result can not be deduced from the usual Katayama duality theorem, since this only works for normal (or reduced) coactions.

Remark 3.3. Now suppose the subgroup $H$ is normal in $G$. Let $\delta^{m} \mid$ denote the restriction of the coaction $\delta^{m}: C^{*}(\mathscr{A}) \rightarrow C^{*}(\mathscr{A}) \otimes C^{*}(G)$ to $G / H$ (see §2). The theory of [5] shows how to induce $\delta^{m} \mid$ to a coaction Ind $\delta^{m} \mid$ of $G$ on an induced $C^{*}$-algebra Ind $C^{*}(\mathscr{A})$ (we refer to $\S 4$ below for the precise definitions), and [5, Theorem 4.1] gives an Ind $C^{*}(\mathscr{A}) \times_{\operatorname{Ind} \delta^{m} \mid} G-$ $C^{*}(\mathscr{A}) \times \delta^{m} \mid G / H$ imprimitivity bimodule $Z$. Dually to the situation regarding Green's imprimitivity theorem for actions, $Z$ is isomorphic to the $C^{*}(\mathscr{A}) \times \delta^{m}$ $G \times \widehat{\delta^{m}} H-C^{*}(\mathscr{A}) \times_{\delta^{m} \mid} G / H$ imprimitivity bimodule of Theorem 3.1. The interested reader can check that the map

$$
\left(a_{s}, t\right) \mapsto\left(a_{s}, s t\right)
$$

of $\mathscr{A} \times G$ extends uniquely to an isomorphism of $X$ onto $Z$ whose left coefficient isomorphism from $C^{*}(\mathscr{A}) \times_{\delta^{m}} G \times \times_{\delta^{m}} H$ onto Ind $C^{*}(\mathscr{A}) \times_{\operatorname{Ind} \delta^{m} \mid} G$ is given on the generators by

$$
\left(a_{s}, t, h\right) \mapsto\left(a_{s}, s t h^{-1} t^{-1}, t h\right) .
$$

Note that in order to see that this map on the generators extends to the desired isomorphism, one uses the fact that $X$ is an $C^{*}(\mathscr{A}) \times \delta^{m} G \times \widehat{\delta}^{m} H-C^{*}(\mathscr{A}) \times{ }_{\delta^{m}}$ $G / H$ imprimitivity bimodule, since then the result follows from an easy application of Lemma 2.9. We actually were not able to get this isomorphism directly, i.e., to deduce Theorem 3.1 directly from [5, Theorem 4.1] in the special case where $H$ is normal in $G$.

In what follows we want to show that the imprimitivity bimodule of Theorem 3.1 factors to an imprimitivity bimodule for the reduced cross-sectional algebras. Thus we want to show

Theorem 3.4. Let $X$ be the $C^{*}(\mathscr{A}) \times{ }_{\delta^{m}} G \times \widehat{\delta^{m}} H-C^{*}(\mathscr{A} \times G / H)$ imprimitivity bimodule of Theorem 3.1, and let I and $J$ denote the kernels 
of the regular representations of $C^{*}(\mathscr{A}) \times \delta_{\delta^{m}} G \times \widehat{\delta^{m}} H$ and $C^{*}(\mathscr{A} \times G / H)$, respectively. Then $I$ is equal to the ideal of $C^{*}(\mathscr{A}) \times \delta_{\delta^{m}} G \times \widehat{\delta}^{m} H$ induced from $J$ via $X$ and therefore $Y:=X /(X \cdot J)$ has a canonical structure as a $C^{*}(\mathscr{A}) \times_{\delta^{m}} G \times \widehat{\delta}^{m}, r, H-C_{r}^{*}(\mathscr{A} \times G / H)$ imprimitivity bimodule.

Recall that a regular representation of $C^{*}(\mathscr{A}) \times \delta_{\delta^{m}} G \times{\widehat{\delta^{m}}} H$ is by definition an induced representation $\operatorname{Ind}_{\{\ell\}}^{H}(\pi \times \mu)$, where $\pi \times \mu$ is any given faithful representation of $C^{*}(\mathscr{A}) \times \delta_{\delta^{m}} G$. Moreover, using Proposition 2.10, we see that the kernel of the regular representation of $C^{*}(\mathscr{A} \times G / H)$ equals $\operatorname{ker}(\pi \times \mu \mid)$, where $\pi \times \mu \mid$ denotes the restriction of $\pi \times \mu$ to $C^{*}(\mathscr{A} \times G / H)$ (note that if we represent $C^{*}(\mathscr{A}) \times \delta^{m} G$ faithfully on a Hilbert space $\mathscr{H}$ via $\pi \times \mu$, then we may identify the pair $(\pi, \mu)$ with the canonical inclusions $\left(j_{C^{*}(\mathscr{A})}, j_{G}\right)$, so that Proposition 2.10 applies). Using these realizations of the (kernels of the) regular representations, and the fact that inducing ideals is compatible with the process of inducing representations, that is, $\operatorname{ker}\left(\operatorname{Ind}^{X}(\pi \times \mu \mid)\right)=$ $\operatorname{Ind}^{X}(\operatorname{ker}(\pi \times \mu \mid))$, where $X$ is the bimodule of Theorem 3.1, Theorem 3.4 follows from

Proposition 3.5. Let $\pi \times \mu$ be any representation of $C^{*}(\mathscr{A}) \times \delta_{\delta^{m}} G$. Then $\operatorname{Ind}_{\{e\}}^{H}(\pi \times \mu)$ is equivalent to $\operatorname{Ind}^{X}(\pi \times \mu \mid)$, where $\operatorname{Ind}^{X}: \operatorname{Rep}\left(C^{*}(\mathscr{A} \times\right.$ $G / H)) \rightarrow \operatorname{Rep}\left(C^{*}(\mathscr{A}) \times \delta_{\delta^{m}} G \times \widehat{\delta^{m}} H\right)$ denotes induction via the bimodule $X$ of Theorem 3.1.

The above proposition constitutes a (new) version of the duality results for induction and restriction of representations obtained in [1], [4], [12] which works for arbitrary subgroups of discrete groups.

Proof of Proposition 3.5. If $(D, H, \alpha)$ is a system with $H$ discrete, then the process of inducing representations from $D$ to $D \times{ }_{\alpha} H$ can be described as follows: let $W_{0}=\Gamma_{c}(D \times H)$, where $D \times H$ denotes the semidirect product bundle. Then translating Green's formulas (see [9]) to this special situation, $W_{0}$ becomes a right pre-Hilbert $D$-module with $D$-valued inner product given by

$$
\langle(b, h),(c, l)\rangle_{D}=\alpha_{h^{-1}}\left(b^{*} c\right) \quad \text { if } h=l \text { (and } 0 \text { else). }
$$

The left convolution action of $\Gamma_{c}(D \times H)$ then extends to a *-homomorphism of $D \times_{\alpha} H$ into $\mathscr{L}_{D}(W)$, where $W$ denotes the Hilbert-module completion of $W_{0}$. The map $\operatorname{Ind}_{\{e\}}^{H}: \operatorname{Rep}(D) \rightarrow \operatorname{Rep}\left(D \times_{\alpha} H\right)$ is just induction via the right-Hilbert $D \times{ }_{\alpha} H-D$ bimodule $W$.

In our situation we have $D=C^{*}(\mathscr{A}) \times_{\delta^{m}} G$ and $\alpha=\widehat{\delta^{m}} \mid$. If we restrict the actions and inner products given above to the dense subalgebras $D_{0}:=$ $\Gamma_{c}(\mathscr{A} \times G)$ of $D$ and $B_{0}:=\Gamma_{c}(\mathscr{A} \times G \times H)$ of $B:=D \times_{\alpha} H$, and the dense $B_{0}-D_{0}$ subbimodule $Y_{0}=\Gamma_{c}(\mathscr{A} \times G \times H)$ of $W$, then $Y_{0}$ becomes a 
pre-right-Hilbert $B_{0}-D_{0}$ bimodule with inner product and actions given by the formulas

$$
\begin{aligned}
\left\langle\left(a_{u}, v, l\right),\left(b_{r}, x, m\right)\right\rangle_{D_{0}} & =\left(a_{u}^{*} b_{r}, x l\right) \quad \text { if } u v=r x, l=m \text { (and } 0 \text { else) } \\
\left(a_{s}, t, h\right) \cdot\left(b_{u}, v, l\right) & =\left(a_{s} b_{u}, v h^{-1}, h l\right) \quad \text { if } t h=u v \text { (and } 0 \text { else) } \\
\left(a_{u}, v, l\right) \cdot\left(b_{y}, z\right) & =\left(a_{u} b_{y}, z l^{-1}, l\right) \quad \text { if } v l=y z \text { (and } 0 \text { else). }
\end{aligned}
$$

Clearly, the completion $Y$ of $Y_{0}$ coincides with $W$ as a right-Hilbert $B-D$ bimodule.

We now describe restriction of representations from $C^{*}(\mathscr{A}) \times{ }_{\delta^{m}} G$ to $C^{*}(\mathscr{A} \times$ $G / H)$ in bimodule language. For this we view $Z:=C^{*}(\mathscr{A}) \times \delta_{\delta^{m}} G$ as a rightHilbert $C^{*}(\mathscr{A} \times G / H)-C^{*}(\mathscr{A}) \times \delta^{m} G$ bimodule equipped with the canonical right $C^{*}(\mathscr{A}) \times \delta_{\delta^{m}} G$-valued inner product and left action of $C^{*}(\mathscr{A} \times G / H)$ given by the natural homomorphism of $C^{*}(\mathscr{A} \times G / H)$ into $M\left(C^{*}(\mathscr{A}) \times \delta_{\delta^{m}} G\right)$. Then the restriction $\pi \times \mu \mid$ is equivalent to the induced representation $\operatorname{Ind}^{Z}(\pi \times \mu)$. If we restrict our attention to the dense subspace $Z_{0}:=\Gamma_{c}(\mathscr{A} \times G)$ of $Z$, and the dense subalgebras $D_{0}=\Gamma_{c}(\mathscr{A} \times G)$ and $C_{0}=\Gamma_{c}(\mathscr{A} \times G / H)$ of $D=C^{*}(\mathscr{A}) \times_{\delta^{m}} G$ and $C=C^{*}(\mathscr{A} \times G / H)$, respectively, then the formulas for the inner product and the left action are given on the generators by

$$
\begin{aligned}
\left\langle\left(a_{u}, v\right),\left(b_{r}, x\right)\right\rangle_{D_{0}} & =\left(a_{u}^{*} b_{r}, x\right) & & \text { if } u v=r x \text { (and } 0 \text { else) } \\
\left(a_{s}, t H\right) \cdot\left(b_{u}, v\right) & =\left(a_{s} b_{u}, v\right) & & \text { if } t H=u v H \text { (and } 0 \text { else). }
\end{aligned}
$$

Finally, let $X_{0}=\Gamma_{c}(\mathscr{A} \times G)$ be the $B_{0}-C_{0}$ pre-imprimitivity bimodule of Theorem 3.1 with formulas given as in Equation (3.2). It is now straightforward to check that the map $V: X_{0} \odot Z_{0} \rightarrow Y_{0}$ defined on the generators by

$$
V\left(\left(a_{s}, t\right) \otimes\left(a_{u}, v\right)\right):=\left(a_{s} a_{u}, u^{-1} t, t^{-1} u v\right) \quad \text { if } t H=u v H \text { (and } 0 \text { else) }
$$

extends to a right-Hilbert $B-D$ bimodule isomorphism of $X \otimes_{C} Z$ onto $Y$. This gives the following chain of unitary equivalences among representations of $C^{*}(\mathscr{A}) \times \delta_{\delta^{m}} G \times \widehat{\delta^{m}} H$ :

$$
\begin{aligned}
\operatorname{Ind}_{\{e\}}^{H}(\pi \times \mu) & \cong \operatorname{Ind}^{Y}(\pi \times \mu) \cong \operatorname{Ind}^{X \times{ }_{C} Z}(\pi \times \mu) \\
& \cong \operatorname{Ind}^{X}\left(\operatorname{Ind}^{Z}(\pi \times \mu)\right) \cong \operatorname{Ind}^{X}(\pi \times \mu \mid) .
\end{aligned}
$$

As an immediate corollary of Theorem 3.4 we get the following application to amenability of Fell bundles in the sense of Exel and Kumjian (see the discussion in Section 2).

Corollary 3.6. Let $\mathscr{A}$ be a Fell bundle over the discrete group $G$ and let $H$ be a subgroup of $G$. Then the following are equivalent: 
(i) $\mathscr{A} \times G / H$ is an amenable Fell bundle over the groupoid $G \times G / H$.

(ii) $\mathscr{A} \times G \times H$ is an amenable Fell bundle over the groupoid $G \times G \times H$.

(iii) The semi direct product bundle $\left(C^{*}(\mathscr{A}) \times \delta^{m} G\right) \times H$ is an amenable Fell bundle over $H$.

In particular, $\mathscr{A}$ is amenable if and only if the double dual bundle $\left(C^{*}(\mathscr{A}) \times \delta^{m}\right.$ $G) \times G($ or $\mathscr{A} \times G \times G)$ is amenable.

Note that in the formulation of the above corollary we could have replaced $\left(C^{*}(\mathscr{A}) \times_{\delta^{m}} G\right) \times H$ with $\left(A \times{ }_{\delta} G\right) \times H$, where $\delta: A \rightarrow A \otimes C^{*}(G)$ is any coaction which has $\mathscr{A}$ as an underlying Fell bundle (see [5, Lemma 2.1]). Just to prevent any misunderstandings: the groupoid structure on $G \times G \times H$ is given by

$$
(s, t, h)(u, v, l)=\left(s u, v h^{-1}, h l\right) \quad \text { if } \quad t h=u v,
$$

which is compatible with Equation (3.1).

We are now going to derive from Theorem 3.1 an imprimitivity theorem which works for any coaction of a discrete group $G$. In order to prepare the statement recall from [5] that if $\delta: A \rightarrow A \otimes C^{*}(G)$ is a coaction of $G$ on $A$, and if $(\mathscr{A}, G)$ denotes the corresponding Fell bundle, then $A$ is a completion of $\Gamma_{c}(\mathscr{A})$ with respect to a $C^{*}$-norm $\|\cdot\|_{v}$ which lies between the norms $\|\cdot\|_{\max }$ and $\|\cdot\|_{\min }$ arising from viewing $\Gamma_{c}(\mathscr{A})$ as a dense subalgebra of $C^{*}(\mathscr{A})$ and $C_{r}^{*}(\mathscr{A})$, respectively. If $N$ is a normal subgroup of $G$, we may restrict $\delta$ to a coaction $\delta \mid=\left(\operatorname{id}_{A} \otimes q\right) \circ \delta: A \rightarrow A \otimes C^{*}(G / N)$, where $q: C^{*}(G) \rightarrow$ $C^{*}(G / N)$ denotes the quotient map. If $\delta$ is normal (that is, $\left.A=C_{r}^{*}(\mathscr{A})\right)$, then it follows from the generalization of Mansfield's imprimitivity theorem to nonamenable groups obtained in [11, Corollary 3.4], that $A \times_{\delta \mid} G / N$ is Morita equivalent to the reduced double crossed product $A \times{ }_{\delta} G \times{ }_{\widehat{\delta}, r} N$, but it was not clear at all whether there is a similar imprimitivity theorem for general coactions. For discrete $G$, the following theorem gives a complete answer to this open question.

Theorem 3.7. Let $(A, G, \delta)$ be a coaction of the discrete group $G$, and let $N$ be a normal subgroup of $G$. Let $(\mathscr{A}, G)$ denote the corresponding Fell bundle, and let $\|\cdot\|_{v}$ denote the $C^{*}$-norm on $\Gamma_{c}(\mathscr{A})$ corresponding to A. Then there exists a $C^{*}$-norm $\|\cdot\|_{\mu}$ (lying between $\|\cdot\|_{\max }$ and $\|\cdot\|_{\min }$ ) on $\Gamma_{c}\left(\left(A \times{ }_{\delta} G\right) \times N\right)$ and a quotient $Z$ of the bimodule $X$ of Theorem 3.1 such that $Z$ becomes an

$$
A \times{ }_{\delta} G \times \widehat{\delta}, \mu N-A \times \times_{\delta \mid} G / N
$$

imprimitivity bimodule, where $A \times \times_{\delta} G \times \widehat{\gamma}_{, \mu} N$ denotes the completion of $\Gamma_{c}\left(\left(A \times_{\delta} G\right) \times N\right)$ with respect to $\|\cdot\|_{\mu}$. Moreover, if $\|\cdot\|_{\nu}$ is the minimal 
(respectively, maximal) norm, then $\|\cdot\|_{\mu}$ is also the minimal (respectively, maximal) norm.

Proof. Let $\delta^{m} \mid$ and $\delta^{n} \mid$ denote the restrictions of the coactions $\delta^{m}$ and $\delta^{n}$ of $G$ on $C^{*}(\mathscr{A})$ and $C_{r}^{*}(\mathscr{A})$ to $G / N$, respectively, and let

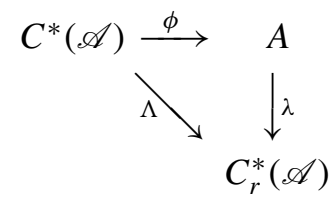

be the commutative diagram of surjections determined by the identity map on $\Gamma_{c}(\mathscr{A})$. Since $\phi, \lambda$, and $\Lambda$ are equivariant with respect to the coactions $\delta^{m}, \delta$, and $\delta^{n}$, and hence also with respect to their restrictions $\delta^{m}|, \delta|$, and $\delta^{n} \mid$, we obtain a commutative diagram of surjections

$$
\begin{aligned}
& C^{*}(\mathscr{A}) \times_{\delta^{m} \mid} G / N \stackrel{\phi \times G / N}{\longrightarrow} A \times_{\delta \mid} G / N \\
& \Lambda \times G / N \searrow \quad \downarrow \searrow \quad \text {. } \\
& C_{r}^{*}(\mathscr{A}) \times{ }_{\delta^{n} \mid} G / N
\end{aligned}
$$

Note that $\phi \times G / N, \lambda \times G / N$, and $\Lambda \times G / N$ are all given by the identity on $\Gamma_{c}(\mathscr{A} \times G / N)$, sitting as a dense subalgebra in all three crossed products. Moreover, it follows from Corollaries 2.8 and 2.12 that the identity map on $\Gamma_{c}(\mathscr{A} \times G / N)$ also induces isomorphisms $C^{*}(\mathscr{A}) \times_{\delta^{m} \mid} G / N \cong C^{*}(\mathscr{A} \times G / N)$ and $C_{r}^{*}(\mathscr{A}) \times_{\delta^{n} \mid} G / N \cong C_{r}^{*}(\mathscr{A} \times G / N)$.

Let $X$ be the $C^{*}(\mathscr{A}) \times{ }_{\delta^{m}} G \times \widehat{\delta^{m}} N-C^{*}(\mathscr{A}) \times{ }_{\delta^{m} \mid} G / N$ imprimitivity bimodule of Theorem 3.1. Since the crossed product $A \times{ }_{\delta} G$ and the dual action $\widehat{\delta}$ only depend on the Fell bundle $\mathscr{A}$ and not on the particular choice of the crosssectional algebra $A$ (see [5, Lemma 2.1]) we can replace $C^{*}(\mathscr{A}) \times{ }_{\delta^{m}} G \times \widehat{\delta^{m}} N$ on the left hand side with either $A \times{ }_{\delta} G \times{ }_{\hat{\delta}} N$ or $C_{r}^{*}(\mathscr{A}) \times \times_{\delta^{n}} G \times \widehat{\delta}^{n} N$. If $\|\cdot\|_{v}=\|\cdot\|_{\max }$, we have $A=C^{*}(\mathscr{A})$ and $\delta=\delta^{m}$, so $X$ is actually an $A \times{ }_{\delta} G \times \widehat{\delta}_{\delta} N-A \times{ }_{\delta \mid} G / N$ imprimitivity bimodule. Thus we get $\|\cdot\|_{\mu}=\|\cdot\|_{\max }$ in this case. If $\|\cdot\|_{\nu}=\|\cdot\|_{\min }$, (that is, $A=C_{r}^{*}(\mathscr{A})$ and $\delta=\delta^{n}$ ), then Theorem 3.4 shows that $X$ factors through an $A \times{ }_{\delta} G \times \widehat{\delta}, r N-A \times{ }_{\delta \mid} G / N$ imprimitivity bimodule, and we get $\|\cdot\|_{\mu}=\|\cdot\|_{\min }$ on the left hand side.

In general, it follows from the above considerations of the maps $\phi \times G / N$, $\lambda \times G / N$ and $\Lambda \times G / N$ that the kernel $L$ of $\phi \times G / N: C^{*}(\mathscr{A}) \times \delta_{\delta^{m} \mid} G / N \rightarrow$ $A \times{ }_{\delta \mid} G / N$ contains the kernel of the regular representation $\Lambda \times G / N$, and hence the ideal $K$ of $A \times{ }_{\delta} G \times \widehat{\delta} N$ induced from $L$ via $X$ contains the kernel of the regular representation of $A \times{ }_{\delta} G \times \widehat{\delta} N$ (which by Theorem 3.4 is the ideal induced from $\operatorname{ker} \Lambda \times G / N$ via $X)$. It follows that $\left(A \times{ }_{\delta} G \times \widehat{\delta}_{\delta} N\right) / K$ is a 
completion of $\Gamma_{c}\left(\left(A \times_{\delta} G\right) \times N\right)$ with respect to a norm $\|\cdot\|_{\mu}$ which lies between $\|\cdot\|_{\max }$ and $\|\cdot\|_{\min }$. Therefore $Z:=X /(X \cdot L)$ is an $A \times_{\delta} G \times \times_{\widehat{\delta}, \mu} N-A \times_{\delta \mid} G / N$ imprimitivity bimodule.

\section{Applications to induced coactions}

Recall from [5] that if $H$ is a normal subgroup of the discrete group $G$ and $\epsilon: D \rightarrow D \otimes C^{*}(G / H)$ is a coaction of the quotient group $G / H$ with underlying Fell bundle $\mathscr{D}$, then the induced coaction $\delta:=\operatorname{Ind} \epsilon$ is defined as the dual coaction on the maximal cross-sectional algebra $C^{*}\left(q^{*} \mathscr{D}\right)$, where $q^{*} \mathscr{D}$ denotes the pull back of $\mathscr{D}$ via the quotient map $q: G \rightarrow G / H$. In [5, Theorem 5.6] we prove an analogue of a theorem of Olesen and Pedersen, in which we show that an action of $G$ is twisted in the sense of Green over a normal subgroup $H$ if and only if the dual coaction is induced from a coaction of $G / H$. Of course it is a natural question whether the dual of this result is also true, namely whether a coaction $\delta$ of $G$ is induced from $G / H$ if and only if the dual action $\widehat{\delta}$ is twisted over $H$. We are now prepared to show that this is indeed the case if we assume the coaction is maximal.

THEOREM 4.1. Let $\delta$ be a coaction of the discrete group $G$ on the $C^{*}$-algebra $A$, and assume that $A=C^{*}(\mathscr{A})$, where $\mathscr{A}$ is the associated Fell bundle. Then $\delta$ is induced from a quotient $G / H$ of $G$ by a normal subgroup $H$ if and only if there exists a Green-twist $\tau: H \rightarrow U M\left(A \times{ }_{\delta} G\right)$ for the dual action $\widehat{\delta}$ of $G$ on $A \times{ }_{\delta} G$.

Proof. Suppose first that $\delta$ is induced from a coaction $\epsilon: D \rightarrow D \otimes$ $C^{*}(G / H)$ of $G / H$. This means that $\mathscr{A}$ is the pull-back bundle $q^{*} \mathscr{D}=\left\{\left(D_{s H}, s\right)\right.$ : $s \in G\}$, where $\mathscr{D}$ is the Fell bundle over $G / H$ corresponding to $\epsilon$. It follows then from [5, Theorem 4.1] that $\widehat{\delta}$ is Morita equivalent to the inflated action Inf $\widehat{\epsilon}$. Since the trivial homomorphism taking $H$ to the identity of $U M\left(D \times{ }_{\epsilon} G / H\right)$ is a twist for Inf $\widehat{\epsilon}$, and since Morita equivalence of actions preserves the property of being twisted over $H$, by [2, Proposition 2], it follows that $\widehat{\delta}$ is twisted over $H$.

Let us now assume that $\widehat{\delta}$ is twisted over $H$. Put $E=A \times_{\delta} G$, and let us look at the semi-direct product bundle $E \times G$ corresponding to $\widehat{\delta}$. The $E \times \widehat{\delta} G-A$ imprimitivity bimodule $X$ of Theorem 3.1 for the case $H=G$ is the completion of the $B_{0}-C_{0}$ pre-imprimitivity bimodule $X_{0}$ with operations given by the corresponding special case of (3.2). For $t \in G$ define $X_{t}=$ $\overline{\operatorname{span}}\left\{\left(A_{s}, t^{-1}\right): s \in G\right\} \subseteq X$. Then the following assertions are true:

(i) $(E, s) \cdot X_{t}=X_{s t}$ and $X_{s} \cdot A_{t} \subseteq X_{s t}$;

(ii) $\left\langle X_{s}, X_{t}\right\rangle_{A} \subseteq A_{s^{-1} t}$ and $\left\langle X_{e}, X_{s}\right\rangle_{A}=A_{s}$ 
for all $s, t \in G$, where all spaces here are to be interpreted as the respective closed linear spans.

Since, by assumption, $\widehat{\delta}$ is twisted over $H$, the semidirect product bundle $E \times G$ is induced from the twisted semidirect product bundle $E \times{ }_{H} G$ over $G / H$, by [5, Theorem 5.6]. Thus, by [5, Theorem 5.1] there exists a map $v: H \rightarrow U M(E \times G)$ with $v_{h} \in U M(E, h)$ satisfying $(b, t) v_{h}=v_{t h t^{-1}}(b, t)$ for all $(b, t) \in E \times G$ and $h \in H$ (see [5, §5] for the notation). If we manage to construct a map $u: H \rightarrow U M(A)$ with similar properties, the result will follow from the other direction of [5, Theorem 5.1].

Since $v_{h}$ is canonically identified with an element of $U M(E \times \widehat{\delta} G)$, it acts as a unitary adjointable operator on the Hilbert $A$-module $X$, with adjoint $v_{h}^{*}=v_{h}^{-1}=v_{h^{-1}}$. Because $X_{s}=(E, e) \cdot X_{s}$ by (i), each $v_{h}$ gives rise to an isometry $x_{s} \mapsto v_{h} \cdot x_{s}$ from $X_{s}$ to $X_{h s}$ with inverse $x_{h s} \mapsto v_{h^{-1}} \cdot x_{s}$. We use this fact to define left and right multiplication of $u_{h}$ with elements in $A_{s}$ :

$u_{h}\left\langle x_{e}, x_{s}\right\rangle_{A}=\left\langle x_{e}, v_{h} \cdot x_{s}\right\rangle_{A} \in A_{h s}$ and $\left\langle x_{e}, x_{s}\right\rangle_{A} u_{h}=\left\langle x_{e}, v_{s h s^{-1}} \cdot x_{s}\right\rangle_{A} \in A_{s h}$.

To see that these formulas determine well-defined isometric maps $A_{s} \rightarrow A_{h s}$ and $A_{s} \rightarrow A_{s h}$, respectively, we first observe that for all $x_{t} \in X_{t}, x_{e} \in X_{e}$ and $x_{s} \in X_{s}$,

$$
\begin{aligned}
x_{t} \cdot\left\langle x_{e}, v_{h} \cdot x_{s}\right\rangle_{A} & ={ }_{B_{0}}\left\langle x_{t}, x_{e}\right\rangle v_{h} \cdot x_{s}=v_{t h t^{-1} B_{0}}\left\langle x_{t}, x_{e}\right\rangle \cdot x_{s} \\
& ={ }_{B_{0}}\left\langle v_{t h t^{-1}} \cdot x_{t}, x_{e}\right\rangle \cdot x_{s}=v_{t h t^{-1}} \cdot x_{t} \cdot\left\langle x_{e}, x_{s}\right\rangle_{A}
\end{aligned}
$$

Using (4.2) for $t=e$ we compute

$$
\begin{aligned}
& \left\|\sum_{i=1}^{n}\left\langle x_{e}^{i}, v_{h} \cdot x_{s}^{i}\right\rangle_{A}\right\|^{2} \\
& =\left\|\sum_{i, j=1}^{n}\left\langle v_{h} \cdot x_{s}^{j}, x_{e}^{j}\right\rangle_{A}\left\langle x_{e}^{i}, v_{h} \cdot x_{s}^{i}\right\rangle_{A}\right\|=\left\|\sum_{i, j=1}^{n}\left\langle v_{h} \cdot x_{s}^{j}, x_{e}^{j} \cdot\left\langle x_{e}^{i}, v_{h} \cdot x_{s}^{i}\right\rangle_{A}\right\rangle_{A}\right\| \\
& \stackrel{(4.2)}{=}\left\|\sum_{i, j=1}^{n}\left\langle v_{h} \cdot x_{s}^{j}, v_{h} \cdot x_{e}^{j} \cdot\left\langle x_{e}^{i}, x_{s}^{i}\right\rangle_{A}\right\rangle_{A}\right\|=\left\|\sum_{i, j=1}^{n}\left\langle v_{h} \cdot x_{s}^{j}, v_{h} \cdot x_{e}^{j}\right\rangle_{A}\left\langle x_{e}^{i}, x_{s}^{i}\right\rangle_{A}\right\| \\
& =\left\|\sum_{i, j=1}^{n}\left\langle x_{s}^{j}, x_{e}^{j}\right\rangle_{A}\left\langle x_{e}^{i}, x_{s}^{i}\right\rangle_{A}\right\|=\left\|\sum_{i=1}^{n}\left\langle x_{e}^{i}, x_{s}^{i}\right\rangle_{A}\right\|^{2} .
\end{aligned}
$$

Thus, left multiplication with $u_{h}$, as defined in Equation (4.1), is a well-defined isometry with inverse $u_{h^{-1}}$, and replacing $h$ by $s h s^{-1}$ gives the similar result for right multiplication. We show $\left(a_{s} u_{h}\right) a_{t}=a_{s}\left(u_{h} a_{t}\right)$ for all $s, t \in G$ and 
$h \in H:$

$$
\begin{aligned}
\left(\left\langle x_{e}, x_{s}\right\rangle_{A} u_{h}\right)\left\langle y_{e}, y_{t}\right\rangle_{A} & =\left\langle x_{e}, v_{s h s^{-1}} \cdot x_{s}\right\rangle_{A}\left\langle y_{e}, y_{t}\right\rangle_{A} \\
& =\left\langle x_{e}, v_{s h s^{-1}} \cdot x_{s} \cdot\left\langle y_{e}, y_{t}\right\rangle_{A}\right\rangle_{A} \\
& =\left\langle x_{e}, B_{0}\left\langle v_{s h s^{-1}} \cdot x_{s}, y_{e}\right\rangle \cdot y_{t}\right\rangle_{A} \\
& =\left\langle x_{e}, v_{s h s^{-1} B_{0}}\left\langle x_{s}, y_{e}\right\rangle \cdot y_{t}\right\rangle_{A} \\
& =\left\langle x_{e}, B_{0}\left\langle x_{s}, y_{e}\right\rangle v_{h} \cdot y_{t}\right\rangle_{A} \\
& =\left\langle x_{e}, x_{s}\right\rangle_{A}\left\langle y_{e}, v_{h} \cdot y_{t}\right\rangle_{A} \\
& =\left\langle x_{e}, x_{s}\right\rangle_{A}\left(u_{h}\left\langle y_{e}, y_{t}\right\rangle_{A}\right) .
\end{aligned}
$$

To see that $u_{h} \in U M\left(A_{h}\right)$ it now suffices to check that $u_{h}^{*}=u_{h^{-1}}\left(=u_{h}^{-1}\right)$. Using (4.2) we first compute

$$
\begin{aligned}
\left(\left\langle x_{r}, x_{t}\right\rangle_{A} u_{h}\right)\left\langle x_{e}, x_{s}\right\rangle_{A} & =\left\langle x_{r}, x_{t} \cdot\left\langle x_{e}, v_{h} \cdot x_{s}\right\rangle_{A}\right\rangle_{A} \\
& =\left\langle x_{r}, v_{t h t^{-1}} \cdot x_{t} \cdot\left\langle x_{e}, x_{s}\right\rangle_{A}\right\rangle_{A} \\
& =\left\langle x_{r}, v_{t h t^{-1}} \cdot x_{t}\right\rangle_{A}\left\langle x_{e}, x_{s}\right\rangle_{A}
\end{aligned}
$$

from which it follows that right multiplication of $u_{h}$ with an inner product $\left\langle x_{r}, x_{t}\right\rangle_{A} \in A_{r^{-1} t}$ is given by the formula $\left\langle x_{r}, x_{t}\right\rangle_{A} u_{h}=\left\langle x_{r}, v_{t h t^{-1}} \cdot x_{t}\right\rangle_{A}$. Using this we now compute

$$
\begin{aligned}
\left(u_{h}\left\langle x_{e}, x_{s}\right\rangle_{A}\right)^{*}=\left\langle x_{e}, v_{h} \cdot x_{s}\right\rangle_{A}^{*} & =\left\langle v_{h} \cdot x_{s}, x_{e}\right\rangle_{A}=\left\langle x_{s}, v_{h^{-1}} \cdot x_{e}\right\rangle_{A} \\
& =\left\langle x_{s}, x_{e}\right\rangle_{A} u_{h^{-1}}=\left\langle x_{e}, x_{s}\right\rangle_{A}^{*} u_{h^{-1}},
\end{aligned}
$$

which proves $u_{h}^{*}=u_{h^{-1}}$. Since it follows directly from the definition of left and right multiplication with $u_{h}$ that $u_{s h s^{-1}} a_{s}=a_{s} u_{h}$ for all $a_{s} \in A_{s}$, we see that $h \mapsto u_{h} \in U M\left(A_{h}\right)$ satisfies all requirements of [5, Theorem 5.1], and the result follows.

\section{REFERENCES}

1. Echterhoff, S., Duality of induction and restriction for abelian twisted covariant systems, Math. Proc. Cambridge Philos. Soc. 116 (1994), 301-315.

2. Echterhoff, S., Morita equivalent twisted actions and a new version of the Packer-Raeburn stabilization trick, J. London Math. Soc. 50 (1994), 170-186.

3. Echterhoff, S., Crossed products with continuous trace, Mem. Amer. Math. Soc. 123 (1996), no. 586.

4. Echterhoff, S., Kaliszewski, S., and Raeburn, I., Crossed products by dual coactions of groups and homogeneous spaces, J. Operator Theory 39 (1998), 151-176.

5. Echterhoff, S. and Quigg, J., Induced coactions of discrete groups on $C^{*}$-algebras, Canad. J. Math. 51 (1999), 745-770.

6. Echterhoff, S. and Raeburn, I., The stabilisation trick for coactions, J. Reine Angew. Math. 470 (1996), 181-215. 
7. Exel, R., Amenability for Fell bundles, J. Reine Angew. Math. 492 (1997), 41-73.

8. Fell, J. M. G. and Doran, R. S., Representations of ${ }^{*}$-Algebras, Locally Compact Groups, and Banach *-Algebraic Bundles, vol. 2, Academic Press, 1988.

9. Green, P., The local structure of twisted covariance algebras, Acta Math. 140 (1978), 191250.

10. Imai, S. and Takai, H., On a duality for $C^{*}$-crossed products by a locally compact group, J. Math. Soc. Japan 30 (1978), 495-504.

11. Kaliszewski, S. and Quigg, J., Imprimitivity for $C^{*}$-coactions of non-amenable groups, Math. Proc. Cambridge Philos. Soc. 123 (1998), 101-118.

12. Kaliszewski, S., Quigg, J., and Raeburn, I., Duality of restriction and induction for $C^{*}$ coactions, Trans. Amer. Math. Soc. 349 (1997), 2085-2113.

13. Kasparov, G. G., Equivariant $K$ K -theory and the Novikov conjecture, Invent. Math. 91 (1988), 147-201.

14. Katayama, Y., Takesaki's duality for a non-degenerate co-action, Math. Scand. 55 (1985), $141-151$.

15. Kumjian, A., Fell bundles over groupoids, Proc. Amer. Math. Soc. 126 (1998), 1115-1125.

16. Mansfield, K., Induced representations of crossed products by coactions, J. Funct. Anal. 97 (1991), 112-161.

17. Quigg, J., Full and reduced $C^{*}$-coactions, Math. Proc. Cambridge Philos. Soc. 116 (1994), $435-450$.

18. Quigg, J., Discrete $C^{*}$-coactions and $C^{*}$-algebraic bundles, J. Austral. Math. Soc. Ser. A 60 (1996), 204-221.

19. Quigg, J. and Spielberg, J., Regularity and hyporegularity in $C^{*}$-dynamical systems, Houston J. Math. 18 (1992), 139-151.

20. Raeburn, I., On crossed products by coactions and their representation theory, Proc. London Math. Soc. 3 (1992), 625-652.

21. Sieben, N., Morita equivalence of $C^{*}$-crossed products by inverse semigroup actions and partial actions, Rocky Mountain J. Math. 31 (2001), 661-686.

WESTFÄLISCHE WILHELMS-UNIVERSITÄT

MATHEMATISCHES INSTITUT

EINSTEINSTR. 62

D-48149 MÜNSTER

GERMANY

E-mail: echters@math.uni-muenster.de
DEPARTMENT OF MATHEMATICS

ARIZONA STATE UNIVERSITY

TEMPE, ARIZONA 85287

USA

E-mail: quigg@math.la.asu.edu 